\title{
CONTROLLABILITY OF TWO-DIMENSIONAL BILINEAR SYSTEMS
}

\author{
Carlos José Braga B., Joao Gonsalves F. and Osvaldo do Rocio \\ Universidade Estadual de Maringá, Brasil \\ Luiz San Martin \\ Universidade Estadual de Campinas, Brasil*
}

\begin{abstract}
For bilinear control systems $\dot{x}=A x+u B x, x \in \mathbf{R}^{2}, A$ and $B$ $2 \times 2$ matrices, necessary and sufficient conditions are given for the controllability on $\mathbf{R}^{2}-\{0\}$. The method is through Lie theory, and follows the program outlined by this theory which consists in finding first the connected subgroups of the group $G l(2)$ of all invertible matrices which are transitive on $\mathbf{R}^{2}-\{0\}$, and then look at the subsemigroups of these subgroups which are transitive. A detailed and nearly self contained exposition of the determination of the transitive subgroups is presented. It turns out that they are $G l^{+}(2), S l(2)$ and the commutative group of nonzero complex numbers. Controllability is analysed by considering these groups separately. In the case of $S l(2)$ the controllability is decided with the aid of a result of [15] about semigroups

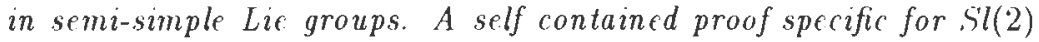
is presented. This case by case analysis recouers the necessary and sufficicnt conditions given by Lepe and Joó and Tuan (see [10]).
\end{abstract}

* Research paritially supported by CNPq Grant No 301060/94-0. 


\section{Introduction}

The controllability problem of control systems proved to be one of the difficult problems in the geometric theory of control systems. Indeed, if for the linear systems there are simple criteria, such as the Kalman rank condition (see for example [20]), for nonlinear systems there are only a few situations where simply verified necessary and sufficient conditions are available. At this regard we mention the already classical results by .Jurdjevic and Sussmann [11], about right invariant systems on compact Lie groups and by Lobry [14] (see also [16]), about systems whose vector fields have a dense set of recurrent points. For these systems the Lie algebra rank condition turns out to be a necessary and sufficient condition for controllability in case it is assumed that the system is analytic. With the development of a geometric theory of semigroups (see the books $[7],[x]$ ). new methods in the study of controllability of invariant systems on Lie groups were discovered. By applying one of these methods Hilgert, Hofmann and Lawson [6] studied controllability on solvable and nilpotent Lie groups, obtaining a necessary and sufficient condition in the latter case. Controllability in nilpotent groups were studied also by Ayala [1], using different methods.

The purpose of this paper is to present a detailed analysis of the controllability of a two dimensional bilinear system

$$
\dot{x}=A x+u B x
$$

with unrestricted control $u \in \mathbf{R}$. Here $x \in \mathbf{R}^{2}$ and $A$ and $B$ are $2 \times 2$ matrices. The controllability of general $d \times d$ bilinear systems were studied elsewhere with partial answers. Sufficient conditions were given in the seminal paper by Jurdjevic and Kupka [12], which were afterwards extended to controllability on semi-simple Lie groups (see $[4,5,13.19]$ ). By piecing together these results with the determination of the transitive groups on $\mathbf{R}^{d}-\{0\}$ (see $[2,3]$ ), further sufficient conditions are achieved. The specific case of two dimensional systems were studied by Lepe and complemented Joó and Tuan (see [10]). Here we take a different route from these authors and analyse the cases according to the Lie algebra generated by the matrices $A$ and $B$. At the final we recover the necessary and sufficient conditions given in [10].

In our approach to controllability, we consider piecewise constant controls. Then it is well known that (1.1) is controllable if and only if the semigroup

$$
S_{\Sigma}=\left\{e^{t_{1} X_{1}} \cdots e^{t_{k} X_{k}}: t_{i} \geq 0, X_{i}=A, \pm B, h^{\prime}>0\right\}
$$


generated by $\Sigma=\{A, \pm B\}$ is transitive on $\mathbf{R}^{2}-\{0\}$, in the sense that for all $x, y \in \mathbf{R}^{2}-\{0\}$ there exists $g \in S_{\Sigma}$ such that $g x=y$ (see e.g. [12]). This being so, a necessary condition for controllability is that the group

$$
C_{\Sigma}=\left\{e^{t_{1} X_{1}} \cdots e^{t_{k} X_{k}}: t_{i} \in \mathbf{R}, X_{i}=A, \pm B, k>0\right\}
$$

generated by $\Sigma$ is transitive on $\mathbf{R}^{2}-\{0\}$. Now, it is a stanclard fact in control theory that $G_{s}$ is a connected Lie subgroup of the group $C$ ill $(2)$ of all invertible $2 \times 2$ matrices and that $S_{\Sigma} \subset$ ( ${ }_{\Sigma}$ has nonempty interior in the intrinsic topology of $G_{\Sigma}$ (see [11]). Moreover, the Lie algebra of $G_{\Sigma}$ is generated by $A$ and $B$. With these facts in mind, our first job is to find the groups which are transitive on $\mathbf{R}^{2}-\{0\}$. Using Lie algebraic arguments, we show that the only connected transitive groups are $\mathrm{Cl}^{+}(2)$, the group of the matrices with positive determinant, the group $S l(2)$ of matrices with determinant one, and $S O(2) \times \mathbf{R}^{+}$which is isomorphic to the group $C^{*}$, of non zero complex numbers. Once we have the groups, we proceed to analyse the semigroups with interior points on them. The case of the group $C^{*}$ presents no difficulty because this group is abelian and diffeomorphic to a cylinder, so one can see almost inmediately the transitive semigroups. Since every invertible matrix is the product of a multiple of the identity matrix times a matrix with determinant one, the case where the group is $G l^{+}(2)$ is reduced to the case where $\left(i_{\Sigma}=S l(2)\right.$. The analysis of this group is the central one. For it, we use a general result proved in [15], which in our context asserts that a semigroup $S \subset S l(2)$ with non void interior coincides with $S l(2)$ if and only if it is transitive on $\mathbf{R} P^{1}$, the set of one dimensional subspaces of $\mathbf{R}^{2}$. With the purpose of turning the paper more self contained, in Section 3 below we adapt the general proof of this result given in [15] to the situation considered here.

Although we consider in this paper only systems with unrestricted controls, it should be noted that most of our methods could be applied also to systems with restricted controls and to other classes of systems such as the discrete-time systems like those studied in [17].

The paper is divided as follows: In Section 2 the groups transitive on $\mathbf{R}^{2}-\{0\}$ are determined. Section 3 is devoted to the proof that no proper subsemigroup with nonempty interior of $S l(2)$ is transitive on the projective line $\mathbf{R} P^{1}$. In Section 5 the controllability of (1.1) is studied with the assumption that $\operatorname{tracot} A=\operatorname{tracotr} B=0$. It is shown that the system is controllable if and only if $\operatorname{det}[A, B]<0$. Sections 6 and 7 consider the cases of the groups $\mathrm{Gl}^{+}(2)$ and $S O(2) \times \mathbf{R}^{+}$respectively. Finally, in Section 8 we recover the necessary and sufficient conditions of Theorems $\mathrm{A}$ and 1 
of $[10]$, with some refinements, by recollecting our preceding work.

\section{Transitive groups}

In this section we find the linear Lie groups which are transitive on $\mathbf{R}^{2}-\{0\}$. The linear groups transitive on $\mathbf{R}^{d}-\{0\}$ were classified in $[2,3]$. We present here an approach which is specific for $d=2$.

Let ( $r$ be a connected Lie subgroup of $(i l(2)$, the group of invertible linear matrices, and denote by $\mathbf{g}$ the Lie algebra of $G$. It is the Lie algebra of those matrices $X$ such that $\exp t X \in G$ for all $t \in \mathbf{R}$. Due to the fact that $\mathbf{R}^{2}-\{0\}$ is connected, a necessary and sufficient condition for $G$ to be transitive is that the orbit

$$
G x=\{g x: g \in G\}
$$

is open for any $x \in \mathbf{R}^{2}-\{0\}$. The tangent space to ( $i x$ at $x$ is given by

$$
\mathbf{g x}=\{\mathbf{A x}: \mathbf{A} \in \mathbf{g}\} .
$$

Hence, $G x$ is open if and only if $\mathbf{g} \mathbf{x}=\mathbf{R}^{\mathbf{2}}$. In the sequel we shall say that $\mathbf{g}$ is transitive provided this condition holds for every $x \in \mathbf{R}^{2}-\{0\}$ so that $G$ is transitive if and only if $\mathbf{g}$ is transitive. Our purpose here is to show that the only transitive Lie algebras and corresponding groups are

1. $\operatorname{gl}(2)$, the Lie algebra of all real $2 \times 2$ matrices, which is the Lie algebra of the connected group $G l^{+}(2)=\{g: \operatorname{det} g>0\}$.

2. $\mathbf{s l}(\mathbf{2})$, the subalgebra of $\mathbf{g l}(\mathbf{2})$ consisting of the zero trace $2 \times 2$ matrices. This is the Lie algebra of $S l(2)$, the group of determinant one matrices.

3. $\mathbf{s o}(\mathbf{2}) \leftrightarrow \mathbf{R} 1$ where so(2) stands for the one dimensional algebra of skew-symmetric matrices and 1 denotes the identity $2 \times 2$ matrix. This is the Lie algebra of the group $S O(2) \times\left(\mathbf{R}^{+} 1\right)$ which consists of a rotation (element of $S O(2)$ ) followed by a homothety (matrix in $\mathbf{R}^{+} 1$ ). The elements of this group are the invertible matrices of the form

$$
\left(\begin{array}{cc}
a & -b \\
b & a
\end{array}\right)
$$

which are in bijection with the non zero complex numbers $a+i b$. So that $S O(2) \times\left(\mathbf{R}^{+} 1\right)$ is isomorphic to $\mathbf{C}^{*}$. This is actually a class 
of transitive groups. Two groups in this class are conjugate to each other by an invertible matrix which is given by the choice of different basis in $\mathbf{R}^{2}$.

It is not hard to cherk that these algebras and groups are indeed transitive on $\mathbf{R}^{2}-\{0\}$. We have

$$
\left(\begin{array}{cc}
x & 0 \\
y & 1 / x
\end{array}\right)\left(\begin{array}{l}
1 \\
0
\end{array}\right)=\left(\begin{array}{l}
x \\
y
\end{array}\right) \text { for } \quad x \neq 0
$$

and

$$
\left(\begin{array}{cc}
0 & -1 / y \\
y & 0
\end{array}\right)\left(\begin{array}{l}
1 \\
0
\end{array}\right)=\left(\begin{array}{l}
0 \\
y
\end{array}\right) \text { for } y \neq 0
$$

so that the orbit of $(1,0)$ under $S l(2)$ is $\mathbf{R}^{2}-\{0\}$. Consequently this group and $G l^{+}(2) \supset S l(2)$ are transitive on $\mathbf{R}^{2}-\{0\}$. The transitivity of $S O(2) \times \mathbf{R}^{+} 1$ follows from

$$
|v|\left(\begin{array}{cc}
\cos \theta & -\sin \theta \\
\sin \theta & \cos \theta
\end{array}\right)\left(\begin{array}{l}
1 \\
0
\end{array}\right)=v
$$

for $v \in \mathbf{R}^{2}-\{0\}$ where $\theta$ is the angle between $v$ and $(1,0)$.

The proof that these are the only transitive groups requires some lemmas. We start with the following well known fact about two dimensional subalgebras of $\mathbf{s l}(\mathbf{2})$.

Lemma 2.1. Let $\mathbf{g} \subset \mathbf{s l}(\mathbf{2})$ be a subalgebra with $\operatorname{dim} \mathbf{g}=\mathbf{2}$. Then there exists a basis $\beta$ of $\mathbf{R}^{2}$ such that the matrices of the linear maps in $\mathbf{g}$ with respect to $\beta$ are of the form

$$
\left(\begin{array}{cc}
a & b \\
0 & -a
\end{array}\right)
$$

Proof. Since $\operatorname{dim} \mathbf{g}=\mathbf{2}, \mathbf{g}$ is solvable. Let us check that $\mathbf{g}$ is not abelian. Put

$$
H=\left(\begin{array}{cc}
1 & 0 \\
0 & -1
\end{array}\right), P=\left(\begin{array}{ll}
0 & 1 \\
0 & 0
\end{array}\right), Q=\left(\begin{array}{ll}
0 & 0 \\
1 & 0
\end{array}\right) \text {. }
$$

These matrices form a basis of $\mathbf{s l}(\mathbf{2})$. Their brackets are given by

$$
[H, P]=2 P, \quad[H, Q]=-2 Q, \quad[P, Q]=H .
$$


Let $X$ and $X^{\prime}$ be such that $\left[X, X^{\prime}\right]=0$ and write $X=a H+b P+c Q$ and $X^{\prime}=a^{\prime} H+b^{\prime} P+c^{\prime} Q$. Then

$$
\left[X, X^{\prime}\right]=\left(b c^{\prime}-c b^{\prime}\right) H+2\left(a b^{\prime}-b a^{\prime}\right) P+2\left(c a^{\prime}-a c^{\prime}\right) Q .
$$

The condition $\left[X, X^{\prime}\right]=0$ implies that $b c^{\prime}=c b^{\prime}, a b^{\prime}=b a^{\prime}$ and $c a^{\prime}=a c^{\prime}$ so that $X$ and $X^{\prime}$ can not be linearly inclependent. This shows that there are no abelian two dimensional subalgebras in $\mathbf{s l}(2)$ so that $\mathbf{g}$ is not abelian.

Hence there is a basis $\left\{X, Y^{Y}\right\}$ of $\mathrm{g}$ such that $[X, Y]=Y$. Also, by the Theorem of Lie on solvable Lie algebras, there exists a basis of $\mathbf{C}^{2}$ such that the complexifications of the elements of $g$ are written in this basis as

$$
\left(\begin{array}{cc}
\lambda & \mu \\
0 & -\lambda
\end{array}\right)
$$

In this representation, the diagonal entries of $Y$ are zero because $[X, Y]=$ $Y$. Therefore, $Y^{2}=0$ so that there exists a basis $\beta$ of is of $\mathbf{R}^{2}$ such that with respect to $\beta, Y$ is upper triangular with zeros on the main diagonal. Using again the fact that $[X, Y]=Y$ one gets quickly that $X$ is also upper triangular with respect to $\beta$ showing the lemma.

The line spanned by the first element in the basis $\beta$ claimed by this lemma is invariant under $\mathrm{g}$. Hence this lemma shows at once that there are no two dimensional transitive subalgebras contained in sl(2). Since one dimensional subalgebras are not transitive (because $\operatorname{dim}(\mathbf{g x}) \leq \operatorname{dim} \mathbf{g}$ ), and $\operatorname{dim} \mathbf{s l}(2)=\mathbf{3}$ we get

Corollary 2.2. $\mathrm{sl}(2)$ is the only of its subalgebras which is transitive.

We look now at the transitive subalgebras which are not contained in sl(2). Every $2 \times 2$ matrix $A$ can be written as

$$
A=\left(A-\frac{\operatorname{tr} A}{2} 1\right)+\frac{\operatorname{tr} A}{2} 1
$$

so that $\mathbf{g l}(\mathbf{2})$ decomposes as

$$
\operatorname{gl}(\mathbf{2})=\operatorname{sl}(\mathbf{2}) \oplus \mathbf{R} \mathbf{1} .
$$

We use the notation $\mathbf{z}=\mathbf{R} \mathbf{1}$ to indicate the center of $\mathbf{g l}(\mathbf{2})$. Because of this decomposition of $\mathbf{g l}(\mathbf{2})$, any subspace $V \subset \mathbf{g l}(\mathbf{2})$ containing $\mathbf{z}$ decomposes as $V=\mathbf{z}(\operatorname{sl}(\mathbf{2}) \cap \mathbf{V})$. With this in mind, we have the following lemma which characterizes the two-dimensional transitive subalgebras which contain $\mathbf{z}$. 
Lemma 2.3. Take $X \in \operatorname{sl}(\mathbf{2})$ and suppose that $\mathbf{g}=\mathbf{R X} \mathbf{z}$ is transitive. Then $\mathbf{g}$ is isomorphic to so( $\mathbf{2})+\mathbf{z}$.

Proof. The assumption that $\mathbf{g}$ is transitive implies that $X \neq 0$ and since $\operatorname{tr} X=0$, its real Jordan canonical form is one of the following

$$
\left(\begin{array}{cc}
a & 0 \\
0 & -a
\end{array}\right),\left(\begin{array}{ll}
0 & 1 \\
0 & 0
\end{array}\right),\left(\begin{array}{cc}
0 & -a \\
a & 0
\end{array}\right) .
$$

In the first two cases the matrices in $\mathbf{g}$ are upper triangular in a swuitable basis of $\mathbf{R}^{2}$ so that $\mathbf{g}$ cannot be transitive. It remains the third case, but then $\mathbf{g}$ is isomorphic to $\mathbf{s o}(\mathbf{2})$ as claimed.

Corollary 2.4. The two dimensional transitive subalgebras are isomorphic to $\mathbf{s o}(\mathbf{2})+\mathrm{z}$.

Proof. Let $g$ be transitive with dimg $=2$. If $g \cap z \neq\{0\}$ then we are in the situation of the above lemma because $\operatorname{din} z=1$. Otherwise, let $\pi$ be the projection of $\mathbf{g l}(2)$ onto $\mathrm{sl}(2)$ according to the decomposition $(2.2)$. Then $\operatorname{dim} \pi(\mathbf{g})=2$ so that the elements of $\pi(\mathbf{g})$ are upper triangular matrices in some basis by Lemma 2.1. This implies that the elements of $\mathbf{g}$ are also upper triangular so that $\mathbf{g}$ is not transitive.

('oncerning the three dimensional subalgebras containing $\mathbf{z}$, we have

Lemma 2.5. Suppose that $\mathbf{g}=\mathbf{h} t \mathbf{z}$ where $\mathbf{h}$ is a subalgebra of $\mathbf{s l}(\mathbf{2})$ of dimension 2 and $\mathrm{z}$ is as above. Then $\mathrm{g}$ is not transitive.

Proof. By Lemma 2.1 there is a basis such that the elements of $\mathbf{h}$ are upper triangular. In this basis, the elements of $\mathbf{g}$ are also upper triangular so that this algebra is not transitive.

Now, let $\mathbf{g}$ be a transitive algebra with $\operatorname{dim} \mathbf{g}=\mathbf{3}$. By the above lemma, $\mathbf{z}$ is not contained in $\mathbf{g}$ and since $\operatorname{dim} \mathbf{z}=\mathbf{1}$ it happens that $\mathbf{g} \cap \mathbf{z}=\{\mathbf{0}\}$ which implies that

$$
\operatorname{gl}(2)=\mathbf{g}() \mathbf{z}
$$

However, $\mathbf{s l}(\mathbf{2})$ is the only subalgebra which complements $z$. In fact, taking the basis $\{H, P, Q\}$ as in (2.1), the fact that $\mathbf{g}$ complements $\mathbf{z}$ ensures the 
existence of reals $h, p$ and $q$ such that $H^{\prime}=H+h 1, P^{\prime}=P+p 1$ and $Q^{\prime}=Q+q \mathrm{l}$ are in $\mathrm{g}$. The brackets between these matrices are

$$
\left[H^{\prime}, P^{\prime}\right]=2 P, \quad\left[H^{\prime}, Q^{\prime}\right]=-2 Q, \quad\left[P^{\prime}, Q^{\prime}\right]=H
$$

so that $\operatorname{sl}(2) \subset \mathbf{g}$, and these algebras coincide because they have the same dimension. We have thus that

Lemma 2.6. $\operatorname{sl}(2)$ is the only three dimensional transitive Lie algebra.

With this lemma we conclude the proof that $\mathbf{g l}(\mathbf{2}), \mathbf{s l}(\mathbf{2})$ and $\mathbf{s o}(\mathbf{2}) \leftrightarrow \mathbf{R}$ are the only transitive subalgebras. In fact, if $\mathbf{g}$ is transitive then $\operatorname{dim} \mathbf{g} \geq \mathbf{2}$. If $\operatorname{dim} \mathbf{g}=\mathbf{2}$ then $\mathbf{g}$ is (isomorphic to) $\mathbf{s o}(\mathbf{2}) \oplus \mathbf{z}$ by Corollary 2.4. The last lemma shows that $\mathbf{g}=\mathbf{s l}(\mathbf{2})$ if $\operatorname{dim} \mathbf{g}=\mathbf{3}$, and it remains only $\mathbf{g l}(\mathbf{2})$ which is four dimensional.

\section{Semigroups in $s l(2)$}

As mentioned in the introduction, the controllability of the control system is equivalent to the transitivity of the semigroup $S_{\Sigma}$ which has nonempty in the connected Lie subgroup $G_{\Sigma}$. We study here the transitivity on $\mathbf{R}^{2}-\{0\}$ of a semigroup $S$ with non void interior in $S l(2)$, which is one of the transitive groups of the previous section.

Denote by $\mathbf{R} P^{1}$ the real one dimensional projective line, which is the set of one dimensional subspaces of $\mathbf{R}^{2}$, and for $v \in \mathbf{R}^{2}-\{0\}$ let $[v]$ be the subspace it spans. We have that $S l(2)$ acts transitively on $\mathbf{R} P^{1}$ by $g[v]=[g v], g \in S l(2), v \in \mathbf{R}^{2}-\{0\}$. A subsemigroup $S$ of $S l(2)$ is said to be transitive on $\mathbf{R} P^{1}$ if for every $u, v \in \mathbf{R}^{2}-\{0\}$ there exists $g \in S$ satisfying $g[u]=[v]$. The main goal of this section is to show that if $S \subset$ $S l(2)$ is a subsemigroup with interior points and transitive on $\mathbf{R} P^{1}$ then it is the whole $S l(2)$. This result is a particular instance of $[15$, Theorem $4.2]$ (see also[18, Theorem 6.2]), and we adapt here the general proof to our specific situation. We start with the following lemma which shows that a proper semigroup cannot contain rotations in its interior.

Lemma 3.1. Let $S \subset S l(2)$ be a semigroup and suppose that there is $X \in \mathbf{s l}(2)$ with purely immaginary eigenvalues, and such that $\exp X$ is in int $S$. Then $S=S l(2)$. 
Proof. The fact that the eigenvalues of $X$ are purely immaginary, imply that its real Jordan form is

$$
\left(\begin{array}{cc}
0 & -a \\
a & 0
\end{array}\right)
$$

so that

$$
\exp (t X)=\left(\begin{array}{cc}
\cos (t a) & -\sin (t a) \\
\sin (t a) & \cos (t a)
\end{array}\right) .
$$

Since $\exp X \in$ int $S$, there exists $t$ such that $\exp (t X) \in$ int $S$ and $t a$ is rational. Putting $h=\exp (t X)$, we have that $h^{n} \in$ int $S$ for every integer $n>0$. However, $h^{n}=1$ for some $n$ because $t a$ is rational. Hence $1 \in i n t S$, which implies that $S=S l(2)$ because in any connected topological group the semigroup generated by a neighborhood of the identity is the whole group.

We show now that proper semigroups of $S l(2)$ do not contain unipotent elements either.

Lemma 3.2. Let $S$ be a subsemigroup of $S l(2)$. Suppose that there exists a nilpotent element $X$ such that $g=\exp X \in$ int $S$. Then $S=S l(2)$.

Proof. If $X=0$ there is nothing to prove. Otherwise the Jordan form of $X$ is

$$
\left(\begin{array}{ll}
0 & 1 \\
0 & 0
\end{array}\right)
$$

Since $g \in$ int $S$ and the exponential map is continuous there exists $\varepsilon>0$ small enough such that

$$
\exp Y=\exp \left(\begin{array}{cc}
0 & 1 \\
-\epsilon & 0
\end{array}\right) \in \operatorname{int}(S) .
$$

The eigenvalues of $Y$ are $\pm i \sqrt{\varepsilon}$ so the claim follows from the previous lemma.

Lemma 3.3. Let $S$ be a subsemigroup of $S l(2)$ with int $S \neq \emptyset$ and assume that $S$ is transitive on $\mathbf{R} P^{1}$. Take $v \in \mathbf{R}^{2}-\{0\}$. Then there exist $w \in \mathbf{R}^{2}$ and $h \in$ ints such that $\{v, w\}$ is a basis and in this basis $h$ is written as

$$
h=\left(\begin{array}{cc}
\mu & 0 \\
0 & \mu^{-1}
\end{array}\right) \text { for some } \mu>0 .
$$


Proof. Take $g \in$ ints such that $g[\eta]=[\eta]$. For its existence, pick $h_{1} \in$ int $S$. Since $S$ is transitive on $\mathbf{R} P^{1}$, there exists $h_{2} \in S$ such that $h_{2} h_{1}[v]=[v]$ so that $g=h_{2} h_{1}$ belongs to int $S$ and fixes [v]. Now, let $u \in \mathbf{R}^{2}$ be such that $\{v, u\}$ is a basis. Since $v$ is an eigenvertor for $g$, in this basis the matrix of $g$ is of the form

$$
g=\left(\begin{array}{cc}
a & b \\
0 & a^{-1}
\end{array}\right)
$$

with $a, b \in \mathbf{R}$ and $a \neq 0$. Clearly, $g^{2} \in$ ints $S$ and its matrix in the basis $\{v, u\}$ is

$$
g^{2}=\left(\begin{array}{cc}
\mu & c \\
0 & \mu^{-1}
\end{array}\right)
$$

with $c \in \mathbf{R}$ and $\mu>0$. If $\mu=1$ then $g^{2}=\exp Y$ with

$$
Y=\left(\begin{array}{ll}
0 & c \\
0 & 0
\end{array}\right)
$$

so Lemma 3.2 implies that $S=S l(2)$, and $S$ certainly contains the claimed diagonal element. Otherwise, let $w=u+c /\left(\mu^{-1}-\mu\right) v$. Than the matrix of $h=g^{2}$ in the basis $\{v, w\}$ has the desired form.

This lemma shows that the assumption that $S$ is transitive on $\mathbf{R} P^{1}$ implies that every vector is an eigenvector of a diagonalizable element in ints. The next lemma improves this by showing that any direction is actually a principal eigenvector for some matrix in int.S. This fact is crucial in the proof of the main result of this section, namely that $S=S t(2)$ if $S$ is transitive on $\mathbf{R} P^{1}$.

Lemma 3.4. With the notations and assumptions of the above lemma take $v \in \mathbf{R}^{2}-\{0\}$. Then there exists $w=0$ and $h \in$ int $S$ in such a way that $\beta=\{v, w\}$ is a basis and the matrix of $h$ with respect $\beta$ is

$$
\left(\begin{array}{cc}
\mu & 0 \\
0 & \mu^{-1}
\end{array}\right)
$$

with $\mu>1$. 
Proof. Let $\{v, w\}$ be as in the last lemma. All the matrices are referred to this basis.

First we observe the following decomposition (Iwasawa decomposition) of $g_{1} \in S l(2)$ which satisfies $g_{1}[v]=[w]$ : There are

$$
h_{1}=\left(\begin{array}{cc}
a & 0 \\
0 & a^{-1}
\end{array}\right) \text { and } n_{1}=\left(\begin{array}{ll}
1 & * \\
0 & 1
\end{array}\right)
$$

such that

$$
g_{1}=\left(\begin{array}{cc}
0 & -1 \\
1 & 0
\end{array}\right) h_{1} n_{1} .
$$

In fact, since $g_{1} v=a w$ for some $a=0$ and det $g_{1}=1$ there is $b \in \mathbf{R}$ such that

$$
g_{1}=\left(\begin{array}{cc}
0 & -a^{-1} \\
a & b
\end{array}\right)
$$

so the claim follows from the following matrix identities $\left(\begin{array}{cc}0 & -a^{-1} \\ a & b\end{array}\right)=\left(\begin{array}{cc}0 & -1 \\ 1 & 0\end{array}\right)\left(\begin{array}{cc}a & b \\ 0 & a^{-1}\end{array}\right)=\left(\begin{array}{cc}0 & -1 \\ 1 & 0\end{array}\right)\left(\begin{array}{cc}a & 0 \\ 0 & a^{-1}\end{array}\right)\left(\begin{array}{cc}1 & \frac{b}{a} \\ 0 & 1\end{array}\right)$.

Analogously, if $g_{2} \in S l(2)$ and $g_{2}[w]=[v]$ we have that $g_{2}$ decomposes after Iwasawa as

$$
g_{2}=h_{2} n_{2}\left(\begin{array}{cc}
0 & 1 \\
-1 & 0
\end{array}\right)
$$

with $h_{2}$ and $n_{2}$ of the same form as $h_{1}$ and $n_{1}$ respectively.

Now we prove the lemma.

Let $h \in$ int $S$ be as in Lemma 3.3. If $\mu>1$ there is nothing to do. Otherwise, we can take $0<\mu<1$ small enough by taking $h^{n}$ instead of $h$ and $\mu^{n}$ instead of $\mu, n \geq 0$ big enough. Since we are assuming that $S$ is transitive on $\mathbf{R} P^{1}$ there are $g_{1}, g_{2} \in S$ such that $g_{1}[v]=[w]$ and $g_{2}[w]=[v]$. We have that $g_{2} h g_{1} \in$ intS because $h \in$ int $S$. Taking Iwasawa decompositions of $g_{1}$ and $g_{2}$ as above we have

$$
g_{2} h g_{1}=h_{2} n_{2}\left(\begin{array}{cc}
0 & 1 \\
-1 & 0
\end{array}\right)\left(\begin{array}{cc}
\mu & 0 \\
0 & \mu^{-1}
\end{array}\right)\left(\begin{array}{cc}
0 & -1 \\
1 & 0
\end{array}\right) h_{1} n_{1} .
$$

Performing the products on the right hand side we get

$$
g_{2} h g_{1}=h_{2} n_{2}\left(\begin{array}{cc}
\mu^{-1} & 0 \\
0 & \mu
\end{array}\right) h_{1} n_{1} .
$$


This equality can be rewritten as $g_{2} h g_{1}=\bar{h} \bar{n}$ with

$$
\bar{h}=h_{2}\left(\begin{array}{cc}
\mu^{-1} & 0 \\
0 & \mu
\end{array}\right) h_{1} \quad \text { and } \quad \bar{n}=\left(\begin{array}{cc}
1 & * \\
0 & 1
\end{array}\right) .
$$

(In fact, if $a$ is a diagonal matrix and $b$ has the same form as $\bar{n}$ above, then $a^{-1} b a$ is like $b$ so that the diagonal matrix can be put on the left by $\left.b a=a\left(a^{-1} b a\right)\right)$.

We have that $\bar{h}$ is the product of three diagonal matrices so it is diagonal. Taking $\mu$ small enough we get

$$
\bar{h}=\left(\begin{array}{cc}
\lambda & 0 \\
0 & \lambda^{-1}
\end{array}\right)
$$

with $\lambda>1$. That is,

$$
g_{2} h g_{1}=\left(\begin{array}{cc}
\lambda & * \\
0 & \lambda^{-1}
\end{array}\right) \in \text { int } S \text { with } \lambda>1 .
$$

Now we can change the basis as in the end of proof of Lemma 3.3 to get the desired diagonal matrix.

As a last comment before the proof of the main theorem of this section we observe the following two facts:

1. Let $(a, b) \subset \mathbf{R}$ be an interval with $b>a>1$. Then there exists $T_{0}>0$ such that $\left(T_{0}, \infty\right) \subset \cup_{n \geq 1}\left(a^{n}, b^{n}\right)$

2. Let $(c, d)$ be an interval with $0<c<d<1$. Then there exists $\varepsilon>0$ such that $(0, \epsilon) \subset \cup_{n \geq 1}\left(c^{n}, d^{n}\right)$

The second fact is a consequence of the first, which follows from $a^{n+1}<$ $b^{n}, n>n_{0}$ for some $n_{0}$, i.e., $\frac{a^{n}}{b^{n}}<\frac{1}{a}$, which follows from $\left(\frac{a}{b}\right)^{n} \rightarrow 0$.

Theorem 3.5. Let $S \subset S l(2)$ be a subsemigroup with interior points. Suppose that $S$ is transitive on $\mathbf{R} P^{1}$. Then $S=\operatorname{Sl}(2)$.

Proof. By Lemma 3.4 there are a basis $\{v, u\}$ of $\mathbf{R}^{2}$ and $h \in$ ints $S$ which is written in this basis as

$$
h=\left(\begin{array}{cc}
\mu & 0 \\
0 & \mu^{-1}
\end{array}\right)
$$


with $\mu>1$. We also have that there exists $g \in$ ints such that $g w=\lambda w$ with $\lambda>1$. In the basis $\{v, w\}$ we can write $g$ as

$$
g=\left(\begin{array}{cc}
\lambda^{-1} & 0 \\
* & \lambda
\end{array}\right)
$$

Since $h, g \in$ int $S$ their powers and powers of their neighborhoods are in int.S. By 1 . above there exists $T_{0}>0$ such that for all $t>T_{0}$

$$
\left(\begin{array}{cc}
t & 0 \\
0 & t^{-1}
\end{array}\right) \in \text { int } S
$$

There is also * such that

$$
\left(\begin{array}{cc}
t^{-1} & 0 \\
* & t
\end{array}\right) \in \text { ints. }
$$

Therefore there exist in the interior of $S$ elements which can be written as

$$
\left(\begin{array}{cc}
t & 0 \\
0 & t^{-1}
\end{array}\right)\left(\begin{array}{cc}
t^{-1} & 0 \\
* & t
\end{array}\right)=\left(\begin{array}{ll}
1 & 0 \\
* & 1
\end{array}\right)=\exp \left(\begin{array}{ll}
0 & 0 \\
* & 0
\end{array}\right) .
$$

and the theorem follows from Lemma 3.2.

In the analysis of the bilinear systems we prove controllability by showing the non existence of a compact invariant subset on $\mathbf{R} P^{1}$ which ensures that $S=S l(2)$ according to the following corollary.

Corollary 3.6. Let $S$ be a subsemigroup of $S l(2)$ with int. $S \neq \emptyset$. Then $S=S l(2)$ if and only if there exists a proper compact subset $C \subset \mathbf{R} P^{1}$, with int $C \neq \emptyset$, which is $S$-invariant, that is, $g C \subset C$ for all $g \in S$.

Proof. For $x \in \mathbf{R} P^{1}$ consider the compact subset $\operatorname{cl}(S x)$ of $\mathbf{R} P^{1}$. We have that $c l(S x)$ is $S$-invariant and there exists a proper compact invariant subset, as in the statement, if and only if $c l(S x)$ is proper for some $x$. Suppose that $\operatorname{cl}(S x)=\mathbf{R} P^{1}$ for every $x \in \mathbf{R} P^{1}$. Then $S$ is transitive on $\mathbf{R} P^{1}$. In fact, the subset

$$
S^{-1}=\left\{g^{-1}: g \in S\right\}
$$

is also a semigroup with nonempty interior in $S l(2)$. Hence for all $y \in \mathbf{R} P^{1}$, $S^{-1} y$ is a subset with non void interior in $\mathbf{R} P^{1}$. Since $S x$ is dense, we have 
that $S^{-1} y \cap S x=\emptyset$. Picking $z \in S^{-1} y \cap S x$, there are $h_{1}, h_{2} \in S$ such that $z=h_{1}^{-1} y$ and $z=h_{2} x$. Therefore $y=h_{1} h_{2} x$ and $S$ is transitive.

Therefore there are no proper compact invariant subsets if and only if $S$ is transitive on $\mathbf{R} P^{\mathbf{1}}$. By the theorem, this last condition is equivalent to $S=S l(2)$.

Remark: An invariant control set is a compact invariant set $C \subset \mathbf{R} P^{\mathbf{1}}$ such that $c l(S x)=C$ for all $x \in C$. It is proved in [15] that there is just one invariant control set. This invariant control set turns out to be the only invariant compact subset.

Another consequence of Theorem 3.5 is that a semigroup $S \subset S l(2)$, with int $S \neq \emptyset$, is transitive on $\mathbf{R}^{2}-\{0\}$ if and only if $S=S l(2)$ because transitivity on $\mathbf{R}^{2}-\{0\}$ implies transitivity on $\mathbf{R} P^{1}$.

\section{Semigroups in $G l^{+}(2)$}

In contrast to $S l(2)$, there are proper semigroups with non void interior in $G^{+}(2)$ which are transitive on $\mathbf{R} P^{\mathbf{l}}$. This happens for instance with the semigroup

$$
\left\{g \in G l^{+}(2): \operatorname{det} g \geq 1\right\} .
$$

In order to look at the semigroups with nonempty interior in $\mathrm{Gl}^{+}(2)$, consider the onto mapping $\pi: G l^{+}(2) \rightarrow S l(2)$ given by

$$
\pi(g)=(\sqrt{\operatorname{det} g})^{-1} g .
$$

This is a homomorphism of groups and a matrix $g \in$ ker $\pi$ if and only if $g=\lambda 1$ for some $\lambda \in \mathbf{R}^{+}$. Thus $\operatorname{ker} \pi$ is isomorphic to the multiplicative group of positive reals. Let $S \subset G l^{+}(2)$ be a semigroup. Then $\pi(S)$ is also semigroup, and int $\pi(S) \neq \emptyset$ in $S l(2)$ in case int $S \neq \emptyset$ in $G l(2)$ because $\pi$ is an open mapping. The following proposition gives information about the semigroups in $G l(2)$ which project onto $S l(2)$.

Proposition 4.1. Let $S \subset G l^{+}(2)$ be a semigroup with int $S \neq \emptyset$, and suppose that $\pi(S)=S l(2)$. Then there exists a semigroup $\Gamma \subset \mathbf{R}^{+}$, with int $\Gamma \neq \emptyset$ such that

$$
S \cap \operatorname{ker} \pi=\Gamma .1 .
$$

Moreover, $S=G l^{+}(2)$ if and only if there are $\lambda_{1}, \lambda_{2} \in \mathbf{R}^{+}$with $\lambda_{1}>1$ and $\lambda_{2}<1$ such that $\lambda_{1} 1$ and $\lambda_{2} 1$ belong to $S$. 
Proof. We have that $S \cap$ ker $\pi$ is a (possibily empty) semigroup because this is an intersection of a semigroup with a group. Hence it is enough to show that it has non void interior. Take $g \in$ ints. Since $\pi\left(S^{\prime}\right)=S l(2)$ there exists $h \in S$ such that $\pi(h)=\pi(g)^{-1}$. We have that $g h \in$ ints and $\pi(g h)=\pi(g) \pi(h)=1$. This shows that int $S \cap$ ker $\pi \neq \emptyset$ which implies that the interior of $S \cap \operatorname{ker} \pi$ is not empty.

For the last statement, we apply the remarks preceding Theorem 3.5. First of all, the existence of $\lambda_{1}>1$ in $\Gamma$ ensures that there is an element bigger than 1 in $i n t \Gamma$. In fact, take $a \in i n t \Gamma$. Then $\lambda_{1}^{n} a \in i n t \Gamma$ for every $n \geq 1$. Taking $n$ big enough, we get $\lambda_{1}^{n} a>1$ in $i n t \Gamma$ as claimed. Now, we have that $\mathbf{R}=\exp \left(\mathbf{R}^{+}\right)$, hence the fact that the interior of $\Gamma$ contains some $b>1$ implies that there exists $T_{0}>1$ such that the interval $\left(T_{0}, \infty\right)$ is contained in int $\Gamma$. Similarly, there exists $T_{1}>1$ such that $\left(0,1 / T_{1}\right) \subset \operatorname{int} \Gamma$. So that there exists $\lambda \in \operatorname{int} \Gamma$ with $\lambda^{-1} \in \operatorname{int} \Gamma$ which shows that $1 \in \operatorname{int} \Gamma$ and $\Gamma=\mathbf{R}^{+}$.

Now, take $g \in G l^{+}(2)$. Then $\pi(g) \in \pi(S)$ so that there exists $h \in S$ such that $g=\lambda h$ for some $\lambda>0$. Since ker $\pi \subset S$ we have that $g \in S$ concluding the proof.

\section{Controllability in the case $G=S l(2)$}

As we have seen the $\operatorname{int}\left(S_{\Sigma}\right)$ is nonempty in $G_{\Sigma}$. In the case of bilinear systems of dimension $2, G_{\Sigma}=S l(2)$ if and only if the Lie algebra generated by $\Sigma=\{A, B\}$ is $\mathbf{s l}(\mathbf{2})$. So we start by looking at this condition.

Lemma 5.1. Take $A, B \in \operatorname{sl}(2)$. Then

1. Suppose that $\operatorname{det} B>0$. Then $\operatorname{det}[A, B] \leq 0$ and the equality holds if and only if $A$ is a multiple of $B$. Moreover, $\{A, B,[A, B]\}$ is linearly independent if and only if $\operatorname{det}[A, B]<0$.

2. Assume that det $B=0$. Then $\operatorname{det}[A, B] \leq 0$ with equality if and only if $[A, B]=\lambda B$. Moreover, the set $\{A, B,[A, B]\}$ is independent if and only if $\operatorname{det}[A, B]<0$.

3. $A$ and $B$ generate $\mathbf{s l}(2)$ if and only if $\operatorname{det}[A, B] \neq 0$. 


\section{Proof.}

1. If $\operatorname{det} B>0$ then in a suitable basis of $\mathbf{R}^{2}$

$$
B=\left(\begin{array}{cc}
0 & -a \\
a & 0
\end{array}\right)
$$

and $B$ is skew-symmetric. Write $A=A_{1}+A_{2}$ with $A_{1}$ skew and $A_{2}$ symmetric. Then $A_{1}$ is a multiple of $B$ so that

$$
[A, B]=\left[A_{2}, B\right] \text {. }
$$

Being the bracket of a skew by a symmetric matrix $[A, B]$ is symmetric, so that $\operatorname{det}[A, B] \leq 0$ because its trace is zero. A direct computation shows that $\left[A_{2}, B\right]$ is not a multiple of $A_{2}$ unless $A_{2}=0$. This implies the independence of the matrices $A, B$ and $[A, B]$.

2. If $\operatorname{det} B=0$ we can assume that

$$
B=\left(\begin{array}{cc}
0 & 1 \\
0 & 0
\end{array}\right) \quad \text { and } \quad A=\left(\begin{array}{cc}
a & b \\
c & -a
\end{array}\right) .
$$

Thus

$$
[A, B]=\left(\begin{array}{cc}
-c & 2 a \\
0 & c
\end{array}\right) .
$$

Therefore $\operatorname{det}[A, B]=-c^{2} \leq 0$ with equality if and only if $[A, B]=$ $2 a B$. From these expressions it is clear that $A, B$ and $C$ are independent if $c \neq 0$.

3. In view of the previous items, it remains to check only the case where $\operatorname{det} A$ and $\operatorname{det} B$ are $<0$. In a suitable basis

$$
A=\left(\begin{array}{cc}
a & 0 \\
0 & -a
\end{array}\right) \quad \text { and } \quad B=\left(\begin{array}{cc}
x & y \\
z & -x
\end{array}\right)
$$

with $-x^{2}-y z<0, a \neq 0$, and we have

$$
[A, B]=\left(\begin{array}{cc}
0 & 2 a y \\
-2 a z & 0
\end{array}\right) .
$$

Hence $\operatorname{det}[A, B] \neq 0$ if and only if $y z \neq 0$. It is readily seen that if $y z=0$ then $A, B$ and $[A, B]$ are triangular so that this set is not independent. On the other hand one checks easily the independence of $A, B$ and $[A, B]$ if $y z \neq 0$. 
This concludes the proof of the lemma.

From this lemma we get immediately the following criteria for checking if the Lie algebra generated by $A$ and $B$ is $\operatorname{sl}(2)$.

Corollary 5.2. Take $A, B \in \mathbf{s l}(\mathbf{2})$. The following conditions are equivalent

1. The Lie algebra generated by $\Sigma=\{A, B\}$ is $\operatorname{sl}(2)$.

2. $A, B$ and $[A, B]$ are linearly independent.

3. $\operatorname{det}[A, B] \neq 0$.

Now, we look at the controllability of bilinear systems of the form $\dot{x}=$ $(A+u B) x$. We are interested in conditions on the matrices $A$ and $B$ which we assume to generate $\mathbf{s l}(\mathbf{2})$. As we have seen in Corollary 3.6 the system is controllable if and only if its semigroup does not leave invariant any proper compact subset of the projective space $\mathbf{R} P^{\mathrm{I}}$. The existence or not of these invariant sets will be detected by looking at the trajectories of the corresponding linear systems. Recall that if $C^{\circ}$ is a matrix with $\operatorname{tr} C=0$, its characteristic polynomial is $x^{2}+\operatorname{det}(C)$ and the eigenvalues are $\pm \sqrt{-\operatorname{det}(C)}$. There are three cases.

1. If $\operatorname{det} C>0$ we can fix a basis such that $C$ is

$$
\left(\begin{array}{cc}
0 & -\lambda \\
\lambda & 0
\end{array}\right)
$$

and the trajectories of the system $\dot{x}=C x$ are circles centered at the origin. In the induced projective action there is just one trajectory.

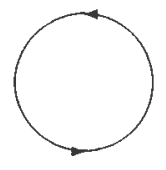

2. If $\operatorname{det} C=0$ with $C=0$ we have, in some basis that

$$
C=\left(\begin{array}{ll}
0 & 1 \\
0 & 0
\end{array}\right)
$$


and the trajectories of the linear system defined by $C$ are the straight lines perpendicular to the $y$-axis and the points in the $x$-axis are stationary points. In the projective action there are two trajectories; a fixed point and a dense trajectory which starts and ends in the fixed point.

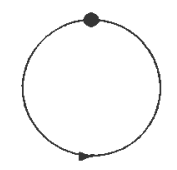

3. If $\operatorname{det} C<0$ we have that

$$
C=\left(\begin{array}{cc}
\lambda & 0 \\
0 & -\lambda
\end{array}\right)
$$

and the trajectories are hyperbolas. The induced projective action consists of two fixed points and two trajectories linking the fixed points.

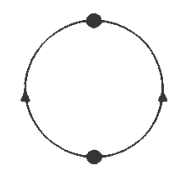

The attractor corresponds to the eigenspace associated with the highest eigenvalue of $C$.

For the controllability of $\dot{x}=(A+u B) x$ we distinguish the cases according to $\operatorname{det} B$. We always assume that $A$ and $B$ are different from 0 .

I If $\operatorname{det} B>0$ and $A \in \operatorname{sl}(2)$ is any matrix with $\operatorname{det}[A, B] \neq 0$ then the system is controllable. This is because the trajectories of $\dot{x}=B x$ are circles and it is not possible to find any compact subset in the projective line which is invariant under the semigroup of the system. Also, Corollary 5.2 ensures that $S_{\Sigma}$ has non void interior.

II If $\operatorname{det} B=0$ and $\operatorname{det}[A, B] \neq 0$ we have controllability again because we can not find any compact and invariant subset of the projective space.

III It remains the case $\operatorname{det} B<0$. There are the possibilities:

1. If $\operatorname{det}(A) \geq 0$ and $\operatorname{det}[A, B] \neq 0$ then the projective trajectories of $A$ are dense and we have controllability. 
2. $\operatorname{det}(A)<0$. In this case the controllability depends on the sign of $\operatorname{det}[A, B]$. Geometrically we have two cases, namely the fixed points $\left\{f_{1}^{A}, f_{2}^{A}\right\}$, in $\mathbf{R} P^{1}$, of $A$ lie in the same trajectory of $B$ or in different trajectories.
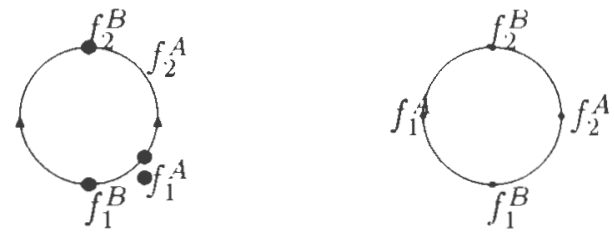

In the first case there is controllability because there is no compact invariant set, and in the second case the system is not controllable. In fact, suppose that $f_{2}^{A}$ is the fixed point of $A$ which is the attractor. Then the closure of the trajectory of $B$ which contains $f_{2}^{A}$ is invariant as show a quick glance at the picture. It should be noted that $\operatorname{det}[A, B] \neq 0$ if and only if the fixed points of $A$ are different from the fixed points of $B$. The coincidence of some of these points imply that $A$ and $B$ have a common eigenvector so that they do not generate $\operatorname{sl}(2)$.

The algebraic meaning of these geometric conditions are as follows. Fix a basis such that

$$
B=\left(\begin{array}{cc}
a & 0 \\
0 & -a
\end{array}\right) \text { and } A=\left(\begin{array}{cc}
\alpha & \beta \\
\gamma & -\alpha
\end{array}\right)
$$

The fixed points of $A$ lie on the same trajectory of $B$ if and only if the eigenvectors of $A$ are in the same quadrant. Now, the eigenvalues of $A$ are $\pm \sqrt{\alpha^{2}+\gamma \beta}$. An eigenvector $(x, y)$ of $A$ satisfies

$$
\left\{\begin{array}{c}
\left(\alpha \pm \sqrt{\alpha^{2}+\gamma \beta}\right) x+\beta y=0 \\
\gamma x+\left(-\alpha \pm \sqrt{\alpha^{2}+\gamma \beta}\right) y=0
\end{array}\right.
$$

Since $y \neq 0$ we can assume that $y=1$ and we get the eigenvectors

$$
\left(x_{ \pm}, 1\right)=\left(-\beta /\left(\alpha \pm \sqrt{\alpha^{2}+\gamma / \beta}\right), 1\right) .
$$

These eigenvectors belong to the same quadrant if and only if $x_{+}$and $x_{-}$have the same sign, and this in turn is equivalent to

$$
-\beta \gamma=\left(\alpha+\sqrt{\alpha^{2}+\gamma \beta}\right)\left(\alpha-\sqrt{\alpha^{2}+\gamma / \beta}\right)>0 .
$$

Since $\operatorname{det}[A, B]=4 a^{2} \gamma \beta$ we have that 
- the system is controllable in case $\operatorname{det}[A, B]<0$, and not controllable if $\operatorname{det}[A, B]>0$.

This concludes the case by case analysis of the systems which generate sil(2). Summarizing, we note the following fact which comes from this analysis.

Theorem 5.3. Let $A, B \in \mathbf{s l}(2)$. Then the semigroup $S_{\Sigma}$ generated by $\Sigma=\{A, \pm B\}$ coincides with $S l(2)$ if and only if $\operatorname{det}[A, B]<0$.

Proof. We have that $S_{\Sigma}=S l(2)$ if and only if $\Sigma$ is controllable on $\mathbf{R}^{2}-\{0\}$ so that it is enough to check that in the above cases controllability occurs if and only if $\operatorname{det}[A, B]<0$. This happens to be the case if det $B<0$ by the last one of the cases. On the other hand, if $\operatorname{det} B \geq 0, \Sigma$ is controllable if and only if $\operatorname{det}[A, B]=0$. By Lemma 5.1 , we have in this case that $\operatorname{det}[A, B] \leq 0$, so the theorem follows.

We turn now to the group $G l(2)$.

\section{The case $G_{\Sigma}=G l^{+}(2)$}

We use the results of the previous section. The idea is to decompose a matrix in $\mathbf{g l}(\mathbf{2})$ as a sum of a matrix in $\mathbf{s l}(\mathbf{2})$ and a diagonal matrix, i.e.

$$
A=\left(A-\frac{\operatorname{tr}(A)}{2} 1\right)+\left(\frac{\operatorname{tr}(A)}{2}\right) 1
$$

We use the notation $\delta(A)=\operatorname{tr}(A) / 2$ and $\tilde{A}=A-\delta(A) 1$. Since the matrices $\delta 1$ and $\tilde{A}$ commute, we have that $[\tilde{A}, \tilde{B}]=[A, B]$ and $e^{t A}=e^{\delta t} e^{t \tilde{A}}$ for every $t \in \mathbf{R}$.

We start by finding a condition ensuring that $A$ and $B$ generate gl(2). Consider the decomposition

$$
\mathbf{g l}(\mathbf{2})=\mathbf{s l}(\mathbf{2}) \oplus \mathbf{R} \mathbf{1}
$$

and note that $A \rightarrow \tilde{A}$ is the associated projection $\mathbf{g l}(\mathbf{2}) \rightarrow \mathbf{s l}(2)$. Recall from Section 2 that if $\mathbf{g} \subset \mathbf{s l}(\mathbf{2})$ is such that its projection $\tilde{\mathbf{g}}=\{\tilde{\mathbf{C}}: \mathbf{C} \in \mathbf{g}\}$ is $\operatorname{sl}(\mathbf{2})$ then $\operatorname{dim} \mathbf{g} \geq \mathbf{3}$ so that $\mathbf{g}=\operatorname{sl}(\mathbf{2})$ or $\operatorname{gl}(\mathbf{2})$. As a consequence, we have

Proposition 6.1. Take $A, B \in \mathbf{g l}(\mathbf{2})$ with $\operatorname{tr}(A) \neq 0$ or $\operatorname{tr}(B) \neq 0$. Then $\operatorname{det}[A, B] \neq 0$ if and only if the Lie algebra generated by $\{A, B\}$ is $\operatorname{gl}(\mathbf{2})$. If this is the case then the Lie algebra generated by $\{\tilde{A}, \tilde{B}\}$ is $\mathbf{s l}(\mathbf{2})$. 
Proof. Let $\mathbf{g}$ be the subalgebra generated by $A$ and $B$. Since det $[A, B]=$ $\operatorname{det}[\tilde{A}, \tilde{B}] \neq 0$, we have from Corollary 5.2 that $\tilde{A}$ and $\tilde{B}$ generate $\mathbf{s l}(\mathbf{2})$. This shows that $\tilde{\mathbf{g}}=\mathbf{s l}(\mathbf{2})$. Therefore the condition that the trace of one of the matrices is not zero implies that $\mathbf{g}=\operatorname{gl}(2)$. Reciprocally, if $\mathbf{g}=\mathbf{g l}(\mathbf{2})$ then the trace of one of the matrices is not zero, otherwise $\mathbf{g}$ would be contained in $\mathbf{s l}(2)$. Also, $\tilde{\mathbf{g}}=\mathbf{s l}(2)$ so that $\operatorname{det}[\tilde{A}, \tilde{B}] \neq 0$ which implies that $\operatorname{det}[A, B] \neq 0$.

Given the system $\Sigma$ determined by $A$ and $B$, denote by $\tilde{\Sigma}$ the system associated with $\tilde{A}$ and $\tilde{B}$. The above proposition shows that $\left(i_{\Sigma}=\operatorname{Sl}(2)\right.$ if the group of $\Sigma$ is $(i l(2)$. Assuming this conclition, a necessary condition for the controllability of $\Sigma$ is the controllability of $\dot{\Sigma}$. In fact, the elements of $S_{\Sigma}$ are products of exponentials like

$$
\epsilon^{C}=e^{\zeta(C)} \epsilon^{\bar{C}} \text {. }
$$

Therefore the elements of $S_{\Sigma}$ are of the form $\lambda g$ with $\lambda>0$ and $g \in S_{\Sigma}$. Since the action of $\lambda g$ on $\mathbf{R} P^{1}$ coincides with the action of $g$, we have that $S_{\tilde{\Sigma}}$ is transitive on $\mathbf{R} P^{\mathbf{l}}$ if $S_{\Sigma}$ is transitive on $\mathbf{R}^{2}-\{0\}$, that is if $\Sigma$ is transitive. Joining together these comments with Theorem 3.5 we get

Proposition 6.2. Suppose that $\{A, B\}$ generates $\mathbf{g l}(\mathbf{2})$. Then a necessary condition for the controllability of $\Sigma$ is that $S_{\bar{\Sigma}}=S l(2)$ and hence that $\dot{\Sigma}$ is controllable.

We look at the controllability of $\Sigma$ by considering the cases in which $\tilde{\Sigma}$ is controllable. This will be made with the aid of Proposition 4.1 and the following

Proposition 6.3. Suppose that $\tilde{\Sigma}$ is controllable and assume that $S_{\Sigma}$ is transitive on a ray $r_{0}$ starting at the origin of $\mathbf{R}^{2}$, that is, for every pair $x, y \in r_{0}$ there exists $g \in S_{\Sigma}$ such that $g x=y$. Then $S_{\Sigma}$ is transitive on $\mathbf{R}^{2}-\{0\}$.

Proof. Since $S_{\tilde{\Sigma}}$ is transitive on $\mathbf{R}^{2}-\{0\}$ it is transitive on the rays, i.e., given two rays $r_{1}$ and $r_{2}$ there exists $g \in S_{\Sigma}$ with $g r_{1}=r_{2}$. But the action of $S_{\Sigma}$ on the rays coincides with that of $S_{\Sigma}$ because the elements of $S_{\Sigma}$ ore of the form $\lambda g, \lambda \in \mathbf{R}^{+}, g \in S_{\bar{\Sigma}}$. Hence $S_{\Sigma}$ is also transitive on the rays. Take $x \in r_{0}$ and let $y \in \mathbf{R}^{2}-\{0\}$ be arbitrarily given. Let $r_{1}$ be the ray which contains $y$. Then there is $g \in S_{\Sigma}$ such that $g r_{1}=r_{0}$ so that 
$g y \in r_{0}$. Hence there exists $h \in S_{\Sigma}$ such that $h g y=x$. Analogously, there is $g_{1} \in S_{\Sigma}$ with $g_{1} r_{0}=r_{1}$. Then $g_{1}^{-1} y \in r_{0}$ and there is $h_{1} \in S_{\Sigma}$ such that $h_{1} x=g_{1}^{-1} y$, i.e., $g_{1} h_{1} x=y$. Therefore $S_{\Sigma}$ is transitive on $\mathbf{R}^{2}-\{0\}$.

We can now consider the different cases. They are divided according to $\operatorname{tr} B$.

I $\operatorname{tr} B=0$. In this case $\Sigma$ is controllable if and only if $\tilde{\Sigma}$ is cortrollable. In fact, suppose $\tilde{\Sigma}$ controllable and distinguish the cases:

1. The eigenvalues of $B$ are real $(\operatorname{det} \tilde{B} \leq 0)$. Then there is an eigenvector $v \in \mathbf{R}^{2}-\{0\}$ and $\exp (t B), t \in \mathbf{R}$ is transitive on the ray spanned by $v$. Therefore Proposition 6.3 implies that $\Sigma$ is controllable.

2. The eigenvalues of $B$ are complex $(\operatorname{det} \tilde{B}>0)$. Let $a \pm i b$ be the eigenvalues. By assumption $a \neq 0 \neq b$. Hence there exists $\bar{t} \in \mathbf{R}$ such that $\bar{t} b=2 k \pi$ for some integer $k=0$. Since in some basis we can write $B$ as

$$
\left(\begin{array}{cc}
a & -b \\
b & a
\end{array}\right),
$$

we have that $\exp (\bar{t} B)=\exp (\bar{t} a) .1$. By taking $-\bar{t}$ instead of $\bar{t}$ we find that $S_{\Sigma}$ satisfies the assumptions of Proposition 4.1. Therefore $S_{\Sigma}=G l(2)$ and $\Sigma$ is controllable.

II $\operatorname{tr} B=0(\tilde{B}=B)$. Then $\operatorname{tr} A \neq 0$. We consider the different possibilities for the Jordan form of $B$.

1. $\operatorname{det} B>0$. Then there is a basis $\beta$ such that the matrix of $B$ is of the form

$$
\left(\begin{array}{cc}
0 & -a \\
a & 0
\end{array}\right)
$$

and the trajectories of $B$ are circles centered at the origin. Denote by $\langle\cdot, \cdot\rangle$ the canonical inner product with respect to $\beta$. The connected subsets which are invariant by the trajectories of $B$ are the rings formed by those $x$ such that $\langle x, x\rangle$ belongs to an interval of $\mathbf{R}^{+}$. Now, $S_{\Sigma} x$ is connected and $B$-invariant for all $x$. Therefore $\Sigma$ is not controllable if and only if $\exp (t A), t \geq 0$ leaves invariant some of these rings. However this condition holds if and only if $A x$ points inwards or outwards some circle 
for every $x$ in the circle, and this is equivalent to the fact that $\langle A x, x\rangle$ does not change sign in the circle. Note that this condition is independent of the length of $x$, so that $\Sigma$ is controllable if and only if $\langle A x, x\rangle$ changes sign in $\mathbf{R}^{2}-\{0\}$. In order to look at these signs, put

$$
S=\frac{A+A^{t}}{2} \text {. }
$$

Then $S$ is symmetric and $\langle A x, x\rangle=\langle S x, x\rangle$. We get thus that a necessary and sufficient condition for the controllability of $\Sigma$ is that $S$ is not semi-definite. Recall that a symmetric matrix is not semi-definite if and only if its eigenvalues are of different sign, that is, if its determinant is negative. Hence we have

- $\Sigma$ is controllable if and only if $\operatorname{det} S<0$.

This condition depends on the basis which puts $B$ in canonical form. We shall translate it into a coordinate free condition. For this use the notations

$$
A=\left(\begin{array}{ll}
a & b \\
c & d
\end{array}\right) \quad X=\left(\begin{array}{cc}
0 & -1 \\
1 & 0
\end{array}\right) .
$$

Then $B=(\sqrt{\operatorname{det} B}) X$ and

$$
[X, A]=\left(\begin{array}{cc}
-b-c & a-d \\
a-d & b+c
\end{array}\right) .
$$

A simple computation shows then that $\operatorname{det} S=\operatorname{det}[X, A]+$ $(\operatorname{tr} A)^{2}$. Substituting $X$ by $B$ in this expression we get

$$
\operatorname{det} S=\frac{1}{\operatorname{det} B}\left(\operatorname{det}[X, A]+\operatorname{det} B(\operatorname{tr} A)^{2}\right) .
$$

Since det $B>0$ we get the following coordinate free condition

- $\Sigma$ is controllable if and only if

$$
\operatorname{det}[X, A]+\operatorname{det} B(\operatorname{tr} A)^{2}<0 .
$$

2. $\operatorname{det} B=0$. In a suitable basis $B$ is given by

$$
\left(\begin{array}{ll}
0 & 1 \\
0 & 0
\end{array}\right)
$$


so that its trajectories are horizontal lines except for the fixed points in the horizontal axis. In analogy with the previous case we find that the connected $B$-invariant subsets are the strips formed by $(s, t)$ with $t$ restricted to an interval of the reals and $s \in \mathbf{R}$. Such a strip is $A$-invariant if and only if $A x$ points upwards or downwards along a line $\{(t, a): t \in \mathbf{R}\}, a$ fixed, and this happens if and only if the second coordinate of $A(t, a)$ does not changes sign when $t$ runs over $\mathbf{R}$. However this coordinate is a linear function of $t$ so that there is no strip invariant under $A$ and $\Sigma$ is always transitive in this case.

3. $\operatorname{det} B<0$. In this case $\Sigma$ is controllable if and only if

$$
\operatorname{det}[A, B]=\operatorname{det}[\tilde{A}, B]<0,
$$

that is, if and only if $\tilde{\Sigma}$ is controllable. In fact, the eigenvalues of $B$ are real so that $\exp (t B)$ is transitive on some ray, therefore the controllability of $\Sigma$ from the controllability of $\tilde{\Sigma}$ follows from Proposition 6.3.

\section{The case with $G_{\Sigma}$ abelian}

We consider here the case where $A$ and $B$ are written simultaneously in the form

$$
\left(\begin{array}{cc}
a & -b \\
b & a
\end{array}\right) .
$$

The Lie algebra generated by these matrices is so(2) $7 \mathbf{R} 1$ if and only if they are linearly independent. The geometry of this case is the easiest one to look at. In fact, as a topological space the group $S O(2) \times(\mathbf{R} 1)$ is a cylinder $S^{1} \times \mathbf{R}$ with the circle $S^{1}$ representing the group $S O(2)$. Also, $S O(2) \times(\mathbf{R} 1)$ is isomorphic to the group $\mathbf{C}^{*}$ whose action on $\mathbf{R}^{2}$ is just multiplication of complex numbers, so that $\Sigma$ is controllable if and only if $S_{\Sigma}$ is the whole group. Since the group is abelian the formula $\exp (X+Y)=\exp X \exp Y$ holds. This implies that $S_{\Sigma}$ is the exponential of the half-plane spanned by $A$ and $B$ :

$$
S_{\Sigma}=\exp \{t A+s B: t \geq 0, s \in \mathbf{R}\} .
$$

Now, the exponential mapping, which is the exponential of complex numbers, is the mapping which rolls up the plane onto the cylinder. Hence $\Sigma$ is controllable if and only if the half-plane spanned by $A$ and $B$ rolls up onto the cylinder. It is clear that this happens if and only if $B$ is not contained in so( 2$)$. Therefore we have: 
- Assuming that $A$ and $B$ are linearly independent, $\Sigma$ is controllable if and only if the eigenvalues of $B$ are not purely immaginary.

Besides the above geometric proof, this fact can be seen also as follows. Suppose that the eigenvalues of $B$ are purely immaginary. Then its trajectories on $\mathbf{R}^{2}$ are circles centered at the origin. Recalling the same situation appearing in Section 6 we have that controllability depends on the sign of the determinant of

$$
S=\frac{A+A^{t}}{2} .
$$

However this matrix is of the form $\lambda .1$ so that det $S=\lambda^{2}>0$ and the system is not controllable. On the other hand, if the eigenvalues of $B$ are not purely immaginary, the half-plane spanned by $A$ and $B$ contains in its interior one of the rays of so(2) so that the interior of this half-plane contains a matrix of the form

$$
\left(\begin{array}{cc}
0 & -b \\
b & 0
\end{array}\right)
$$

with $b=2 k \pi, k$ integer. Therefore $1 \in$ int $S_{\Sigma}$ so that $S_{\Sigma}=S O(2) \times\left(\mathbf{R}^{+} 1\right)$, and the system is controllable.

\section{Conclusions}

We summarize now the previous analysis in the form of necessary and sufficient polynomial conditions.

- Controllability is equivalent to $\operatorname{det}[A, B]<0$ in each one of the following cases:

1. det $B \leq 0$ and $B=0$.

2. det $B>0$ and the eigenvalues of $B$ are real and $B \neq c .1$.

3. $\operatorname{tr} A=\operatorname{tr} B=0$.

The case where the matrices belong to $\mathbf{s l}(2)$ were discussed in Section 5 . As to the other cases, if $\operatorname{det} B \leq 0$ then $B$ is not a multiple of the identity and its eigenvalues are not complex. Therefore controllability holds only if $[A, B] \neq 0$ which means that the Lie algebra generated by $\{A, B\}$ is $\operatorname{sl}(2)$ or $\mathbf{g l}(2)$. By the discussion in Section 6 , we have controllability if and only if $\operatorname{det}[A, B]=\operatorname{det}[\tilde{A}, \tilde{B}]<0$ and the eigenvalues of $B$ are not purely 
immaginary. Hence we get the equivalent condition in case $\operatorname{det} B \leq 0$. This argument applies also to the second case.

If $B$ heal) eigenvalues then $\operatorname{det}[A, B] \leq 0$. So we have the following refinement of the first two cases.

- Controllability is equivalent to $\operatorname{det}[A, B] \neq 0$ in case $B$ has just one eigenvalue and $B \neq c .1$.

In any one of the above situations the group associated with the system is not abelian. This might happen to be the case in the remaining ones which complement the first and second cases above.

- If $B=k .1$ then controllability holds if and only if the eigenvalues of $A$ are complex.

- If the eigenvalues of $B$ are complex and not purely immaginary (so that det $B>0$ and $\operatorname{tr} B \pm 0$ ) then there is controllability if and only if $A$ is ltiple of $B$.

- If the eigenvalues of $B$ are purely immaginary, controllability is equivalent to

$$
\operatorname{det}[A, B]+\operatorname{det} B(\operatorname{tr} A)^{2}<0 .
$$

In this last case, $\operatorname{det}[A, B] \leq 0$ for any $A$ with equality if and only if $[A, B]=0$. If this happens, we are in the situation of Section 7 . Otherwise, the condition is always fulfilled if $\operatorname{tr} A=0$, so we have

- If the eigenvalues of $B$ are purely immaginary and $\operatorname{tr} A=0$ then controllability is equivalent to $[A, B] \neq 0$.

This list covers all the possibilities for $A$ and $B$ providing necessary and sufficient conditions for the controllability of (1.1). 


\section{References}

[1] Ayala B., V.: Controllability of nilpotent systems. Cieometry of nonlinear rontrol and differential inclusions. Banach Center Publications 32, Polish Academy of Sciences (1995).

[2] Boothby, W.: A transitive problem from control theory. Journal of Differential Equations, 17, pp. $296-307,(1975)$.

[3] Boothby, W. and Wilson E.N.: Determination of transitivity of bilinear systems. SIAM Journal on Control and Optimization, 17, pp. $212-221$, (1979).

[4] El Assoudi, R. and J.P. Gauthier: Controllability of right invariant systems on real simple Lie groups of type $F_{4}, G_{2}, C_{n}^{\prime}$ and $B_{n}$. Math. of Control, Signals and Systems, 1, pp. $293-391,(1988)$.

[5] Gauthier, J.P., I. Kupka and G. Sallet: Controllability of right invariant systems on real simple Lie groups. Systems \& Control Letters, 5, pp. $187-190,(1984)$.

[6] Hilgert, J., K.H. Hofmann and J. Lawson: Controllability of systems on a nilpotent Lie group. Beiträge Algebra Geometrie, 30, pp. $185-$ 190, (1985).

[7] Hilgert, J., K.H. Hofmann and J. Lawson: Lie groups, convex cones and semigroups. Oxford University Press (1989).

[8] Hilgert, J. and K.-H. Neeb: Lie semigroups and their applications. Lecture Notes in Mathematics, 1552 Springer Verlag (1993).

[9] Joó, I. and N.M. Tuan: On controllability of bilinear systems I (controllability in finite dimensions). Ann. Univ. Sci. Budapest, 35, pp. $189-215,(1992)$.

[10] - On controllability of bilinear systems II (controllability in two dimensions). Ann. Univ. Sci. Budapest, 35, pp. 217 - 265, (1992).

[11] Jurdjevic, V. and H.J. Sussmann: Control systems on Lie groups. J. of Diff. Eq., 12, pp. $313-329$, (1972). 
13x Carlos Braga, Joao Goncalvez, Osvaldo do Rocio y Luiz San Martin

[12] Jurdjevic, V. and I. Kupka: Control systems subordinated to a group action: Accessibility. J. Diff. Eq., 39, pp. $180-211,(1981)$.

[13] - Control systems on semisimple Lie groups and their homogeneous spaces. Ann. Inst. Fourier (Grenoble), 31, pp. $151-179$, (1981).

[14] Lobry, L.: Controllability of nonlinear systems on compact manifolds. SIAM J. on Control and Optim., 12, pp. 1 - 4, (1974).

[15] San Martin, L.: Invariant control sets on flag manifolds. Math. of Control, Signals and Systems, 6, pp. $41-61,(1993)$.

[16] - Controllability of families of measure preserving vector fields. Systems \& Control Letters, 8, pp. $459-462$, (1987).

[17] - On global controllability of discrete-time control systems. Math. of Control, Signals and Systems, 8, pp. $279-297,(1995)$

[18] San Martin, L.A.B. and P.A. Tonelli: Senigroup actions on homogeneous spaces. Semigroup Forum, 50, pp. $59-88,(1995)$.

[19] Silva Leite, F. and P.E. Crouch: Controllability on classical Lie groups. Math. of Control, Signals and Systems, 1, pp. $31-42$, (1988).

[20] Sontag, E.D.: Mathematical control theory. Springer Verlag (1990).

Received : April 22, 1996 
Carlos José Braga Barros

João Ribeiro Gonçalvez Filho

Osvaldo Germano do Rocío

Departamento de Matemática

Universidade Estadual de Maringá

Cx. Postal 331

87.020-900 Maringá - Pr

Brasil

and

Luiz A. B. San Martín

Instituto de Matemática

Universidade Estadual de Campinas

Cx Postal 6065

13081-970 Campinas - SP

Brasil 